\title{
Toward Patient-Tailored Perfusion Thresholds for Prediction of Stroke Outcome
}

\author{
A. Eilaghi, C.D. d'Esterre, T.Y. Lee, R. Jakubovic, J. Brooks, R.T.-K. Liu, L. Zhang, R.H. Swartz, and R.I. Aviv
}

\begin{abstract}
BACKGROUND AND PURPOSE: Multiple patient-specific clinical and radiologic parameters impact traditional perfusion thresholds used to classify/determine tissue outcome. We sought to determine whether modified baseline perfusion thresholds calculated by integrating baseline perfusion and clinical factors better predict tissue fate and clinical outcome.
\end{abstract}

MATERIALS AND METHODS: CTP within 4.5 hours of acute anterior circulation stroke onset and 5- to 7-day MR imaging were performed for 203 patients with stroke, divided into derivation $(n=114)$ and validation $(n=89)$ data bases. Affected regions were operationally classified as infarct and noninfarct according to baseline CTP and follow-up FLAIR imaging. Perfusion thresholds were derived for each of the infarct and noninfarct regions, without and with transformation by baseline clinical and radiologic variables by using a general linear mixed model. Performance of transformed and nontransformed perfusion thresholds for tissue fate and 90-day clinical outcome prediction was then tested in the derivation data base. Reproducibility of models was verified by using bootstrapping and validated in an independent cohort.

RESULTS: Perfusion threshold transformation by clinical and radiologic baseline parameters significantly improved tissue fate prediction for both gray matter and white matter $(P<.001)$. Transformed thresholds improved the 90 -day outcome prediction for $C B F$ and time-to-maximum $(P<.001)$. Transformed relative CBF and absolute time-to-maximum values demonstrated maximal $\mathrm{GM}$ and WM accuracies in the derivation and validation cohorts (relative CBF GM: 91\%, 86\%; WM: 86\%, 83\%; absolute time-to-maximum 88\%, 79\%, and 80\%, 76\% respectively).

CONCLUSIONS: Transformation of baseline perfusion parameters by patient-specific clinical and radiologic parameters significantly improves the accuracy of tissue fate and clinical outcome prediction.

ABBREVIATION: Tmax = time-to-maximum

T hrombolytic therapy remains the mainstay of acute stroke therapy. ${ }^{1}$ CTP may distinguish hypoperfused but potentially salvageable tissue from irreversibly infarcted tissue ${ }^{2}$ by using single or combinations of perfusion variables. ${ }^{3}$ However, in addition to these perfusion parameters, multiple baseline clinical and radiologic parameters are also significantly associated with tissue outcome, including age, ${ }^{4}$ sex,${ }^{5}$ time-to-scan, ${ }^{6}$ NIHSS score, ${ }^{4}$ hypoattenuation extent or ASPECTS, ${ }^{7}$ rtPA administration, ${ }^{4}$ hyperglycemia, ${ }^{8}$ collateral score, and clot burden score. ${ }^{9}$ Many studies

Received February 14, 2013; accepted after revision May 31

From the Robarts Research Institute (A.E., C.D.d., T.Y.L.), University of Western Ontario, London, Ontario, Canada; and Department of Medical Imaging (A.E., R.J., J.B., R.T.-K.L., R.I.A.), Odette Cancer Centre (L.Z.), and Department of Neurology (R.H.S.), Sunnybrook Health Sciences Centre, University of Toronto, Toronto, Ontario, Canada.

Please address correspondence to Richard I. Aviv, MD, Sunnybrook Health Sciences Centre, 2075 Bayview Ave, Room AG 31, Toronto, ON, M4N 3M5, Canada; e-mail: richard.aviv@sunnybrook.ca

三 Indicates article with supplemental on-line tables.

http://dx.doi.org/10.3174/ajnr.A3740 intuitively show that consideration of baseline clinical parameters together with imaging features in multivariable models better predicts final infarct and outcome status. ${ }^{10,11}$ These predictive models do not provide a practical way to integrate the multiple baseline findings with the perfusion parameter thresholds used for classifying tissue fate, leaving the clinician to balance a combination of factors before deciding on treatment. Integration of this information is crucial to assist clinicians in determining the best treatment strategies for individual patients. For example, a patient with euglycemia and good collaterals may tolerate a lower CBF reduction without infarction better than a patient with hyperglycemia without collaterals. Therefore, using a single CBF threshold will overestimate final infarction. We exploited a derivation CTP data base $(n=114)$, including 90 -day clinical outcome, to calculate, on a per-patient basis, the relative impact of multiple important baseline clinical and radiologic factors on baseline perfusion parameters to define tissue outcome. We used a general linear model for each perfusion variable with region fate (infarct versus noninfarct) as the outcome. The model then cal- 
culated a transformed perfusion parameter on the basis of the relative impact of each clinical variable on the baseline perfusion parameter for the defined outcome in the derivation $(n=114)$ and validation $(n=89)$ datasets. The result was a patient-specific perfusion variable transformation that integrates the important clinical and radiologic data for each region. The purpose of this study was to determine whether these transformed patient-specific models better predict tissue fate and clinical outcome than a nontransformed model.

\section{MATERIALS AND METHODS \\ Study Design and Patient Cohort}

The study was approved by the local research ethics board. Patients included were consecutive tertiary stroke center emergency department admissions, assessed by a stroke neurologist, presenting within 4.5 hours of stroke symptoms, undergoing a CT stroke protocol, and demonstrating an anterior circulation vessel occlusion. Follow-up MR imaging was performed at 5-7 days. Baseline clinical data (ie, age, sex, NIHSS score, rtPA treatment, and cardiovascular risk factors) were collected by a stroke neurologist (with 4 years' experience). Informed consent was obtained from all patients or legal decision-makers. Patients were randomly divided into derivation and validation cohorts. In total, 252 eligible patients were screened. Patients from whom consent could not be obtained $(n=13)$ and those with a posterior circulation stroke ( $n=24)$ and MR imaging contraindications ( $n=12$ cases) were not included. The final patient derivation and validation cohorts consisted of 114 and 89 patients, respectively.

\section{Scan Protocol}

CT stroke imaging was performed on a 64-section CT scanner (VCT; GE Healthcare, Milwaukee, Wisconsin), including the following: pre- (NCCT) and postcontrast CT head scans $(120 \mathrm{kVp}$, $300 \mathrm{~mA}$, 1-second rotation, 5-mm-thick sections, 5-mm space); and baseline and 24-hour CT angiography (0.7-mL/kg iodinated contrast agent up to a maximum $90 \mathrm{~mL}$ [iohexol, Omnipaque, 300- mg Iodine/mL; GE Healthcare, Piscataway, New Jersey], 5to 10 -second delay, $120 \mathrm{kVp}, 270 \mathrm{~mA}, 1$-second/rotation, 1.25 $\mathrm{mm}$-thick sections, table speed of $20.62 \mathrm{~mm} /$ rotation). CTP was performed from the basal ganglia to the lateral ventricles $(80 \mathrm{kVp}$, $150 \mathrm{~mA}, 8 \times 5 \mathrm{~mm}, 1$ second per rotation). Iodinated contrast agent at $0.5 \mathrm{~mL} / \mathrm{kg}$ (maximum, $50 \mathrm{~mL}$ ) was injected at $5 \mathrm{~mL}$ per second $^{-1}$ at 3-5 seconds before sequence start. Cerebral blood flow and volume, mean transit time, and time-to-maxium (Tmax) maps were calculated as previously described by using delay-corrected CTP 4 (GE Healthcare). Follow-up MR imaging was performed at 5-7 days on all patients and minimally included DWI (8125 ms/min [TR/TE]; FOV, 26 cm; image matrix, $128 \times$ 128 pixels; section thickness, $5 \mathrm{~mm}$ with no gap) and FLAIR (8000/120/200 [TR/TE/TI]; FOV, 22 cm; matrix, $320 \times 224$; section thickness, 5; 1-mm gap).

\section{Image Analysis}

Baseline imaging was scored blinded to outcome and included ASPECTS, ${ }^{12}$ clot burden score for thrombus extent, and collateral score for collateral flow. ${ }^{9}$ Recanalization was classified as present or absent, comparing the baseline and 24-hour CTA as previously described. ${ }^{9}$ Infarct was delineated on FLAIR imaging by using Medical Image Processing, Analysis and Visualization, Version 4.4.1 (Center for Information Technology, National Institutes of Health, Bethesda, Maryland). All baseline and follow-up imaging assessments were performed by the same neuroradiologist (R.I.A; with 7 years' experience). FLAIR imaging and baseline CTP were coregistered with NCCT, and the appropriate transformation matrices were applied to each region of interest by using SPM8 (Wellcome Department of Imaging Neuroscience, London, UK). A brain tissue probability map template (Montreal Neurological Institute) was registered to the baseline NCCT by using SPM8. GM and white matter tissue binary masks were generated by creating tissue probability maps within SPM8. Final ROIs were created by intersecting the traced regions with GM and WM masks. A mirror region was created and segmented to provide normal GM and WM regions for relative calculations. Final infarct was defined as the intersection between the MTT perfusion abnormalities and the follow-up MR imaging. Noninfarct regions comprised MTT perfusion abnormality outside the final infarct. To minimize the contribution of blood vessels, we excluded pixels with $\mathrm{CBF}>100 \mathrm{~mL} \times 100 \mathrm{~g}^{-1} \times \min ^{-1}$ or $\mathrm{CBV}>8 \mathrm{~mL} \times 100$ $\mathrm{g}^{-1} \cdot{ }^{13}$

\section{Statistics}

Mean and SD were calculated for age and time-to-scan; median and interquartile ranges for ASPECTS, clot burden score, collateral score, and NIHSS score; and proportions for sex, recanalization, and hyperglycemia. For each patient, mean CBF, CBV, MTT, and Tmax values were calculated from infarct and noninfarct ROIs representing nontransformed baseline parameters. To account for within-subject variability and for unbalanced repeated measures, we used a general linear mixed model, ${ }^{14}$ previously used in the context of tissue outcome prediction in human ${ }^{15}$ and animal ${ }^{16}$ stroke models. Individual patients were considered as random effects; the region, as a fixed effect; and compound symmetry, as a covariance structure. Multiple baseline clinical and radiologic covariates were examined by univariate analysis including age, sex, time-to-scan, rtPA treatment status ( 1 = yes; $0=$ no), hyperglycemia ( $1=$ yes; $0=$ no), NIHSS score, ASPECTS, clot burden score, and collateral score. Significant (ie, $P<.05$ ) or important (ie, $P<.2$ ) baseline covariates were then used to transform mean regional (infarct and noninfarct) absolute and relative baseline perfusion parameters as follows:

Transformed regional mean perfusion parameter $=$ Original Mean Perfusion Parameter $+\beta X_{\beta}$, where $\beta$ and $X_{\beta}$ are vectors of covariates and associated coefficients. ROC analysis was then performed for each of the nontransformed and transformed perfusion parameters to determine the optimal thresholds best predicting final infarct-versus-noninfarct status, similar to prior threshold-based studies. For each nontransformed (model A) and transformed (model B) threshold, the accuracy for tissue fate prediction was then derived. The accuracy was defined as $(T P+T N) /$ $(P+N)$, where $T P, T N, P$, and $N$ are true-positive, true-negative, all positive (ie, infarct) and all negative (ie, noninfarct). Performance of the nontransformed and transformed thresholds was evaluated with Akaike Information Criterion $\left(\mathrm{AIC}=L_{R E S}+2 \mathrm{~K}\right)$. A lower Akaike Information Criterion indicates a better model fit, 
where $L_{R E S}$ represents the restricted maximized $-2 \times \log$ likelihood $(-2 \mathrm{~L})$ of the model, and $k$, the number of parameters in the model. The $\mathrm{G}^{2}$ likelihood ratio statistic is the difference between $-2 \mathrm{~L}$ of the fitted model (transformed threshold) and the reference model (nontransformed threshold). A 2-sided $P$ value was obtained from the $\mathrm{G}^{2}$ likelihood ratio $\chi^{2}$ test. Effect size was calculated for the mean difference divided by the pooled SD between 2 groups of patients by using nontransformed model (model A) and transformed model (model B). To test the reproducibility of the models, we used bootstrapping with unrestricted random sampling. ${ }^{17}$ We drew 1000 bootstrap samples from the derivation dataset. For each bootstrap sample, the general linear mixed model was conducted with clinical covariates (model B). For each clinical covariate, 1000 estimates of coefficient were calculated. The mean and standard error of coefficient estimates were calculated for each clinical covariate.

Cochran-Mantel-Haenszel statistics and 1-way analysis of variance for unbalanced sample sizes were used for group comparisons. Logistic regression was used to determine whether the transformed model (model B) better predicted the clinical outcome than the nontransformed model (model A) by using a dichotomized 90-day modified Rankin score, where mRS $\leq 2$

\begin{tabular}{|c|c|c|c|c|}
\hline In All Patients & $\begin{array}{l}\text { Cutoff } \\
\text { Value }\end{array}$ & Accuracy & AIC & $P$ Value \\
\hline \multicolumn{5}{|l|}{ CBF-GM-Rel } \\
\hline A) Without any covariate & 0.64 & 0.88 & 2465.9 & \\
\hline B) With covariates & 0.78 & 0.91 & 2401.4 & $<.0001^{\mathrm{b}}$ \\
\hline \multicolumn{5}{|l|}{ CBF-WM-Rel } \\
\hline A) Without any covariate & 0.80 & 0.85 & 2693.6 & \\
\hline B) With covariates & 0.87 & 0.86 & 2593.6 & $<.0001^{\mathrm{b}}$ \\
\hline \multicolumn{5}{|l|}{ Tmax-GM-Abs } \\
\hline A) Without any covariate & 6.0 & 0.84 & 1377.8 & \\
\hline B) With covariates & 4.4 & 0.88 & 1358.5 & $.0113^{\mathrm{b}}$ \\
\hline \multicolumn{5}{|l|}{ Tmax-WM-Abs } \\
\hline A) Without any covariate & 5.9 & 0.79 & 1251.5 & \\
\hline B) With covariates & 3.7 & 0.80 & 1232.1 & $.0108^{\mathrm{b}}$ \\
\hline
\end{tabular}

Note:-Rel indicates relative to the contralateral side; Abs, absolute value; AIC, Akaike Information Criterion.

${ }^{a}$ Remaining parameters are presented in On-line Table 2

b Significant.

Table 2: Performance of selected nontransformed (model A) and covariate transformed (model B) perfusion thresholds for tissue fate prediction in derivation and validation data bases

\begin{tabular}{|c|c|c|c|c|c|c|}
\hline \multirow[b]{2}{*}{ Perfusion Parameter } & \multicolumn{3}{|c|}{ Derivation Data Base } & \multicolumn{3}{|c|}{ Validation Data Base } \\
\hline & Sens. & Spec. & Acc. & Sens. & Spec. & Acc. \\
\hline \multicolumn{7}{|l|}{ CBF-GM-Rel } \\
\hline A) Without any covariate & 0.88 & 0.89 & 0.88 & 0.72 & 1 & 0.82 \\
\hline B) With covariates & 0.89 & 0.93 & 0.91 & 0.78 & 1 & 0.86 \\
\hline \multicolumn{7}{|l|}{ CBF-WM-Rel } \\
\hline A) Without any covariate & 0.86 & 0.84 & 0.85 & 0.76 & 0.95 & 0.82 \\
\hline B) With covariates & 0.90 & 0.80 & 0.86 & 0.79 & 0.91 & 0.83 \\
\hline \multicolumn{7}{|l|}{ Tmax-GM-Abs } \\
\hline A) Without any covariate & 0.80 & 0.92 & 0.84 & 0.70 & 0.93 & 0.78 \\
\hline B) With covariates & 0.84 & 0.96 & 0.88 & 0.69 & 0.97 & 0.79 \\
\hline \multicolumn{7}{|l|}{ Tmax-WM-Abs } \\
\hline A) Without any covariate & 0.76 & 0.85 & 0.79 & 0.70 & 0.88 & 0.76 \\
\hline B) With covariates & 0.80 & 0.80 & 0.80 & 0.64 & 0.94 & 0.76 \\
\hline
\end{tabular}

Note:-Rel indicates relative to the contralateral side; Abs, absolute value; Sens, sensitivity; Spec, specificity; Acc, accuracy. was defined as a good outcome. The entropy $R^{2}$ was used to compare model A and model B. $R^{2}$ equals $\left(L_{O}-L_{M}\right) / L_{O}$, where $L_{O}$ and $L_{M}$ represent the maximized $-2 \mathrm{~L}$ of the null model and the fitted model A or model B, respectively. All calculations were performed by using SAS, Version 9.2 (SAS Institute, Cary, North Carolina).

\section{RESULTS}

No significant differences for baseline variables were seen between the derivation and validations cohorts (On-line Table 1). In derivation $(n=114)$ /validation $(n=89)$ cohorts, $74(65 \%) / 61$ patients $(69 \%)$ had $>1$ vessel segment occlusion with a total $n$ of 42/25 ICA, 108/58 MCA-M1, 81/67 M2, 12/9 M3, and 9/9 M4 occlusions. All measured perfusion parameters were expectedly different between the infarct and noninfarct $(P<.001)$ regions. Similarly, significantly different regional CBF and CBV thresholds existed for GM and WM $(P<.001)$ but not for Tmax and MTT $(P>$.326). Therefore subsequent analysis considered GM and WM separately.

GM and WM relative CBF and absolute Tmax best discriminated infarct from noninfarct tissue designations, both in models without and with baseline covariates (Table 1). Effect size for relative CBF in GM and WM was 2.18 and 1.62, and for Tmax in GM and $\mathrm{WM}$, it was 1.46 and 1.26. The remaining transformed and nontransformed cutoff thresholds for infarct and noninfarct tissue classification are presented in On-line Table 2. The Akaike Information Criterion and $\mathrm{G}^{2}$ likelihood statistic improved significantly for both GM and WM for transformed thresholds compared with nontransformed thresholds, indicating significantly better goodness-of-fit for all perfusion parameters except for absolute CBV in WM $(P=.12)$. Transformed thresholds were superior to nontransformed thresholds for prediction of infarct region from the noninfarct region in the derivation and validation cohorts (Table 2). Maximal accuracies for GM were $91 \%$ and $86 \%$ and for WM were $86 \%$ and $83 \%$ in the derivation and validation cohorts, respectively. Figure 1 shows predictions with and without transformation and compares them with the follow-up MR images for the patient.

The transformed absolute Tmax threshold and relative CBF WM represented the most robust overall discriminators of good clinical outcome (Table 3). Results for all perfusion parameters are provided in Online Table 3. Transformed absolute and relative thresholds for CBV were not significantly improved relative to the nontransformed threshold model for GM or WM. Bootstrapping samples showed the reproducibility of the covariate models with an average (SD) decrease in the standard error of $46 \%$ (12\%) (On-line Table 4).

\section{DISCUSSION}

Transformation of baseline perfusion thresholds with patient-specific baseline clinical and radiologic parameters significantly improves goodness-of-fit and ac- 

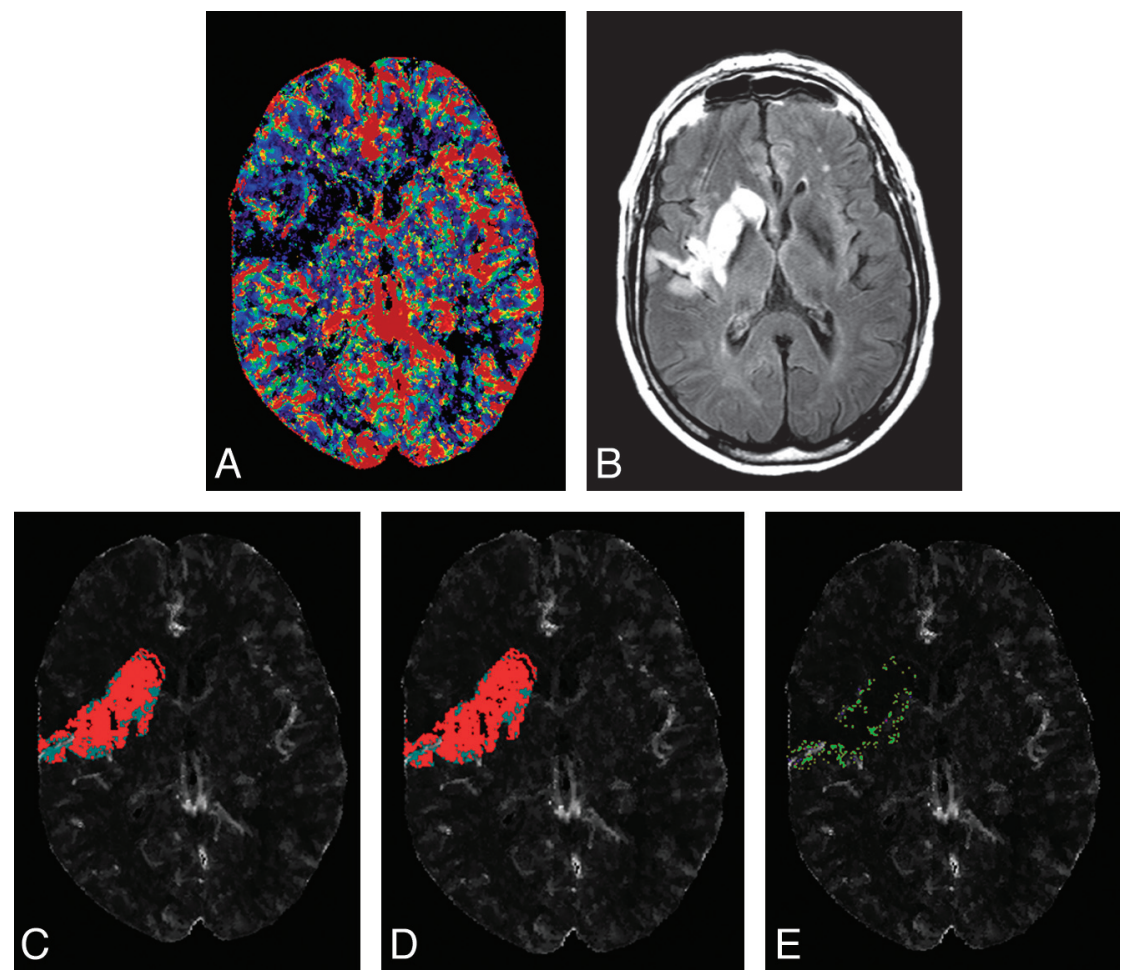

FIG 1. Admission CBF $(A)$ and 7-day follow-up FLAIR (B) of a 74-year-old man with an ICA and MI branch occlusion scanned 168 minutes after symptom onset and administered intravenous rtPA. The region of abnormality on follow-up FLAIR is coregistered to CBF and thresholded on the basis of without $(C)$ and with covariate $(D)$ thresholds from Table 1. Red voxels present predicted infarct, and cyan voxels present predicted noninfarct tissues on $C$ and $D$. E, A subtraction of $C$ and $D$ representing voxels that were designated as infarcted tissue (green) and noninfarcted (pink, very few mainly located adjacent to blood vessels on $E$ ) only on the model with covariates.

Table 3: Selected model performance without and with clinical covariates for good clinical outcome prediction ${ }^{\mathrm{a}}$

\begin{tabular}{|c|c|c|c|}
\hline In All Patients & AIC & $\mathrm{G}^{2}$ & $P$ Value \\
\hline \multicolumn{4}{|l|}{ CBF-GM-Rel } \\
\hline Null model & 291.5 & - & \\
\hline A) Without any covariate & 291.7 & 1.796 & .1803 \\
\hline B) With covariates & 291.3 & 2.247 & .1338 \\
\hline \multicolumn{4}{|l|}{ CBF-WM-Rel } \\
\hline Null model & 282.5 & - & \\
\hline A) Without any covariate & 280.4 & 4.080 & .0434 \\
\hline B) With covariates & 268.9 & 15.613 & $<.0001^{\mathrm{b}}$ \\
\hline \multicolumn{4}{|l|}{ Tmax-GM-Abs } \\
\hline Null model & 291.5 & - & \\
\hline A) Without any covariate & 291.3 & 2.255 & .1332 \\
\hline B) With covariates & 285.4 & 8.116 & $.0044^{b}$ \\
\hline \multicolumn{4}{|l|}{ Tmax-WM-Abs } \\
\hline Null model & 282.5 & - & \\
\hline A) Without any covariate & 280.9 & 3.643 & .0563 \\
\hline B) With covariates & 266.2 & 18.328 & $<.0001^{\mathrm{b}}$ \\
\hline
\end{tabular}

Note:-Rel indicates relative to the contralateral side; Abs, absolute value; AIC, Akaike Information Criterion; $G^{2}$, the difference between $-2 L$ of the fitted model (transformed threshold) and the reference model (nontransformed threshold).

${ }^{a}$ Remaining parameters are presented in On-line Table 3.

b Significant.

curacy for final infarct prediction in derivation and validation cohorts. Transformed CBF, Tmax, and MTT thresholds demonstrated improved prediction of good clinical outcome compared with a standard threshold approach. The best discriminator of infarction was transformed relative CBF followed by Tmax. A bootstrap analysis confirmed the reproducibility of covariates for infarct and noninfarct tissue determination. Expected thresholds were demonstrated for GM compared with WM $(P<$ .001) for all regions of interests.

The observations in the present study have clear and practical clinical implications. An emerging role of advanced stroke imaging is to use hemodynamic data to characterize tissue and estimate the relative proportion of "at-risk" tissue to tissue infarction. Prior studies emphasized the need for parameters that more accurately classify tissue fate. The present study used a novel approach to improving the accuracy for correct tissue characterization by integrating clinically relevant factors with baseline perfusion imaging. Our results show improved tissue characterization and clinical outcome prediction with this approach. Better tissue classification is essential to better facilitate targeted patient selection for thrombolytic therapy.

Use of a single perfusion parameter to characterize tissue is appealing, allowing quick tissue characterization. Automated patient identification as beneficial targets for reperfusion therapy based on a ratio of core-to-penumbral tissue by using Tmax is shown with the RAPID software (Stanford Stroke Centre, Palo Alto, California) applied to MR imaging. ${ }^{18}$ However, the selection algorithm requires DWI for core identification, unavailable on CT. Singleparameter use also does not consider the multitude of other clinical factors that are present within a given patient that impact perfusion threshold techniques. Our findings show that Tmax and CBF thresholds modified by patient-specific parameters may be used to distinguish infarct from noninfarct tissue. These modified thresholds can similarly be used to generate ratios in a fashion similar to RAPID to identify patients most likely to benefit from therapy. The ideal single-perfusion parameter or parameter combination ${ }^{13}$ predicting tissue fate remains uncertain. Among studied parameters, CBF best predicted final infarct consistent with other studies. ${ }^{19,20}$ The reduced performance of CBV for infarct determination in the present study is consistent with prior studies. ${ }^{20}$ This result is intuitive if we consider that CBV reduction may represent already infarcted tissue with minimal prospects for improvement irrespective of other clinical factors such as collaterals and rtPA treatment. ${ }^{21}$

Prognostic models incorporating both baseline clinical and radiologic parameters have previously been proposed. ${ }^{22}$ Imaging parameters, including clot burden score, the Boston Acute Stroke Imaging Scale and CBV ASPECTS; ${ }^{9}$ clot burden and collateral scores; ${ }^{9}$ baseline CT features with an admission NIHSS score, ${ }^{23}$ and ASPECT scoring of CTP maps, ${ }^{24}$ improve prediction of stroke outcome models. The current study is the first to use baseline clinical and radiologic parameters to transform baseline per- 
fusion thresholds by a general linear mixed model and compare the performance solely of the transformed threshold against the conventional threshold for tissue fate and clinical outcome prediction. While clinical factors were used to transform the perfusion threshold, it was only the transformed threshold performance that was compared against the nontransformed threshold for outcome prediction. While the threshold transformations appear relatively modest, they improved not only the accuracy of tissue fate prediction but also prediction of good clinical outcome. The entropy ( $R^{2}$ parameter in On-line Table 3$)$ for transformed models, though small in value ${ }^{25}$ provided significant improvement in clinical outcome predictions in most studied perfusion parameters. These results underscore the contribution of such parameters and confirm a modest but significant modulation of traditional perfusion thresholds. Inclusion of such information is intuitive and considers patient-specific physiology. For example, patients with good baseline collateral supply are more likely to sustain tissue-at-risk even under extreme perfusion conditions compared with patients with poor collateral supply and less severe hypoperfusion. ${ }^{26}$ Therefore, lower thresholds than those in patients with poor collateral supply may be needed in patients with good supply to cause infarction. The transformed thresholds make allowances for such circumstances.

The current study has limitations. The reported perfusion thresholds in this study apply to those centers using similar acquisition and postprocessing techniques. ${ }^{20}$ This limitation is true of every perfusion study and does not imply nongeneralizability. Although thresholds may vary by center, it is reasonable to assume that similar transformation of baseline perfusion thresholds will be achieved by inclusion of baseline clinical and radiologic parameters. DWI is the preferred technique for acute infarct detection but becomes less sensitive within the subacute period ${ }^{27}$ compared with FLAIR sensitivity, which progressively increases. No significant DWI and FLAIR mean lesion volume differences were reported beyond 12 hours. ${ }^{27}$ Strong correlation between final infarct and 5-day FLAIR volume was reported with a tendency toward slight overestimation. ${ }^{28}$ Use of a 5- to 7-day FLAIR for final infarct determination is a pragmatic measure for clinical stroke research and is also consistent with the methodology of several prior publications ${ }^{13}$; most recently Diffusion and Perfusion Imaging Evaluation for Understanding Stroke Evolution II (DEFUSE II). ${ }^{29}$

Recanalization $^{30}$ and reperfusion ${ }^{31}$ statuses, though among the strongest surrogates for clinical outcome, are not available at stroke presentation and are not included in the covariate model. This approach is similar to that in the DEFUSE selection model, whereby the intent is to create a baseline model for optimal patient selection through accurate tissue classification. Recanalization and reperfusion are, however, strongly associated with several important baseline parameters included in the model. For example, IV rtPA treatment, highly correlated with recanalization, is included, thereby considering the intent of the neurologist to administer thrombolytic therapy at baseline. Similarly clot burden score, age, and collateral score are available at baseline and strongly correlate with recanalization. ${ }^{9}$ Further variations of a model including recanalization, reperfusion index, ${ }^{31}$ and NIHSS score change could be developed that may be applied at later time points. While these models may also improve tissue fate and clinical outcome prediction, they will not have the clinical utility of a baseline tool as presented. The improved prediction for tissue fate and clinical outcome was achieved by transformed thresholds solely on the basis of baseline parameters, in the absence of recanalization status, and adds validity to the proposed model as a tool to guide baseline decision-making at a time when the recanalization status is not yet known.

\section{CONCLUSIONS}

In summary, transformation of baseline perfusion thresholds with clinical and radiologic parameters improves the accuracy of tissue fate and clinical outcome prediction over existing threshold methods. Modified thresholds may facilitate more accurate and patient-specific outcome prediction. Greater accuracy could potentially improve patient selection and individualize stroke therapy based on modified perfusion maps.

Disclosures: Ting-Yim Lee-RELATED: Grant: Heart and Stroke Foundation of Canada*; UNRELATED: Royalties: GE Healthcare.* Richard H. Swartz-UNRELATED: Grants/Grants Pending: Heart and Stroke Foundation of Canada*; Payment for Lectures (including service on Speakers Bureaus): Sanofi-Aventis, Bristol Myers Squibb, Comments: Advisory Board (Bristol Myers Squibb). Richard I. AvivRELATED: Grant: MITACS.* *Money paid to the institution.

\section{REFERENCES}

1. Konstas AA, Wintermark M, Lev MH. CT perfusion imaging in acute stroke. Neuroimaging Clin N Am 2011;21:215-38, ix

2. Kamalian S, Konstas AA, Maas MB, et al. CT perfusion mean transit time maps optimally distinguish benign oligemia from true "atrisk" ischemic penumbra, but thresholds vary by postprocessing technique. AJNR Am J Neuroradiol 2012;33:545-49

3. Dani KA, Thomas RG, Chappell FM, et al. Computed tomography and magnetic resonance perfusion imaging in ischemic stroke: definitions and thresholds. Ann Neurol 2011;70:384-401

4. Kwiatkowski TG, Libman RB, Frankel M, et al. Effects of tissue plasminogen activator for acute ischemic stroke at one year. $N$ Engl J Med 1999;340:1781-87

5. De Silva DA, Ebinger M, Davis SM. Gender issues in acute stroke thrombolysis. J Clin Neurosci 2009;16:501-04

6. Adams HP Jr, Adams RJ, Brott T, et al. Guidelines for the early management of patients with ischemic stroke: a scientific statement from the Stroke Council of the American Stroke Association. Stroke 2003;34:1056-83

7. Sillanpaa N, Saarinen JT, Rusanen H, et al. The clot burden score, the Boston Acute Stroke Imaging Scale, the cerebral blood volume ASPECTS, and two novel imaging parameters in the prediction of clinical outcome of ischemic stroke patients receiving intravenous thrombolytic therapy. Neuroradiology 2012;54:663-72

8. Bruno A, Biller J, Adams HP Jr, et al. Acute blood glucose level and outcome from ischemic stroke. Neurology 1999;52:280-84

9. Tan IY, Demchuk AM, Hopyan J, et al. CT angiography clot burden score and collateral score: correlation with clinical and radiologic outcomes in acute middle cerebral artery infarct. AJNR Am J Neuroradiol 2009;30:525-31

10. Saposnik G, Fang J, Kapral MK, et al. The iScore predicts effectiveness of thrombolytic therapy for acute ischemic stroke. Stroke 2012;43:1315-22

11. Fargen KM, Chaudry I, Turner RD, et al. A novel clinical and imaging based score for predicting outcome prior to endovascular treatment of acute ischemic stroke. J Neurointerv Surg 2013;5(suppl 1):i38-i43

12. Barber PA, Demchuk AM, Zhang J, et al. Validity and reliability of a quantitative computed tomography score in predicting outcome of hyperacute stroke before thrombolytic therapy: ASPECTS Study 
Group-Alberta Stroke Programme Early CT Score. Lancet 2000;355:1670-74

13. Murphy BD, Fox AJ, Lee DH, et al. Identification of penumbra and infarct in acute ischemic stroke using computed tomography perfusion-derived blood flow and blood volume measurements. Stroke 2006;37:1771-77

14. Cnaan A, Laird NM, Slasor P. Using the general linear mixed model to analyse unbalanced repeated measures and longitudinal data. Stat Med 1997;16:2349-80

15. Wu O, Koroshetz WJ, Ostergaard L, et al. Predicting tissue outcome in acute human cerebral ischemia using combined diffusion- and perfusion-weighted MR imaging. Stroke 2001;32:933-42

16. Wu O, Sumii T, Asahi M, et al. Infarct prediction and treatment assessment with MRI-based algorithms in experimental stroke models. J Cereb Blood Flow Metab 2007;27:196-204

17. Efron B. Bootstrap methods: another look at the jackknife. Ann Stat $1979 ; 7: 1-26$

18. Lansberg MG, Lee J, Christensen S, et al. RAPID automated patient selection for reperfusion therapy: a pooled analysis of the Echoplanar Imaging Thrombolytic Evaluation Trial (EPITHET) and the Diffusion and Perfusion Imaging Evaluation for Understanding Stroke Evolution (DEFUSE) Study. Stroke 2011;42:1608-14

19. Campbell BC, Christensen S, Levi CR, et al. Cerebral blood flow is the optimal CT perfusion parameter for assessing infarct core. Stroke 2011;42:3435-40

20. Kamalian S, Maas MB, Goldmacher GV, et al. CT cerebral blood flow maps optimally correlate with admission diffusion-weighted imaging in acute stroke but thresholds vary by postprocessing platform. Stroke 2011;42:1923-28

21. Wintermark M, Flanders AE, Velthuis B, et al. Perfusion-CT assess- ment of infarct core and penumbra: receiver operating characteristic curve analysis in $\mathbf{1 3 0}$ patients suspected of acute hemispheric stroke. Stroke 2006;37:979-85

22. Reid JM, Gubitz GJ, Dai D, et al. Predicting functional outcome after stroke by modelling baseline clinical and CT variables. Age Ageing 2010;39:360-66

23. Lam WW, Leung TW, Chu WC, et al. Hyperacute extensive middle cerebral artery territory infarcts: role of computed tomography in predicting outcome. J Comput Assist Tomogr 2004;28:650-53

24. Kloska SP, Dittrich R, Fischer T, et al. Perfusion CT in acute stroke: prediction of vessel recanalization and clinical outcome in intravenous thrombolytic therapy. Eur Radiol 2007;17:2491-98

25. Cohen J. Statistical Power Analysis for the Behavioral Sciences. Hillside, New Jersey: Lawrence Erlbaum Associates; 1987

26. Liebeskind DS. Collateral circulation. Stroke 2003;34:2279-84

27. van Everdingen KJ, van der Grond J, Kappelle LJ, et al. Diffusionweighted magnetic resonance imaging in acute stroke. Stroke 1998;29:1783-90

28. Gaudinski MR, Henning EC, Miracle A, et al. Establishing final infarct volume: stroke lesion evolution past $\mathbf{3 0}$ days is insignificant. Stroke 2008;39:2765-68

29. Diffusion Weighted Imaging Evaluation for Understanding Stroke Evolution Study-2 (DEFUSE-2). ClinicalTrials.gov. http://clinicaltrials. gov/show/NCT01349946. Accessed May 11, 2012

30. Rha JH, Saver JL. The impact of recanalization on ischemic stroke outcome: a meta-analysis. Stroke 2007;38:967-73

31. Soares BP, Tong E, Hom J, et al. Reperfusion is a more accurate predictor of follow-up infarct volume than recanalization: a proof of concept using CT in acute ischemic stroke patients. Stroke 2010; 41:e34-40 